\title{
Sitagliptin versus saxagliptin in decompensated type 2 diabetes mellitus patients
}

\author{
Antonio Asti, ${ }^{1}$ Alessandra D'Alessandro, ${ }^{2}$ Francesco Paolo Zito, ${ }^{2}$ Salvatore Nardi, ${ }^{1}$ Giovanni Sarnelli, ${ }^{2}$ \\ Giorgio Maresca, ${ }^{1}$ Giuseppe D’Alessandro ${ }^{1}$
}

${ }^{1}$ Department of Clinical Medicine and Emergency, S. Maria di Loreto Nuovo Hospital, Napoli; ${ }^{2}$ Department of Clinical Medicine and Surgery, Gastroenterology Unit, Federico II University, Napoli, Italy

\begin{abstract}
Sitagliptin and saxagliptin are oral hypoglycemic agents inhibitors of DPP-4, indicated in the treatment of type 2 diabetes mellitus in combination with metformin, in patients who have not achieved adequate glycemic control. In our study we enrolled 128 decompensated type 2 diabetes patients while on metformin maximum dosage. At time 0' we have detected, body mass index (BMI), total cholesterol, high- and low-density lipoproteins (HDL and LDL), triglycerides, transaminases and pancreatic amylase; patients were randomized to receive sitagliptin or saxagliptin; follow-up was performed after 4 months with the revaluation of the same variables and adverse events. In both sitagliptin and saxagliptin groups we observed a significant reduction in fasting glucose, glycated hemoglobin, weighing, BMI, triglycerides, while the reduction in total cholesterol, LDL cholesterol did not reach statistical significance. There was no suspension of therapy, adverse events appeared minor and temporary. In conclusion, our observations highlight the almost identical efficacy of sitagliptin and saxagliptin. These data reinforce even more the idea that we should think about this class of drugs as the next step in patients failing therapy with metformin.
\end{abstract}

\section{Introduction}

Type 2 diabetes mellitus (T2DM) is a chronic and progressive disease, characterized by persistent

Correspondence: Alessandra D’Alessandro, Department of Clinical Medicine and Surgery, Gastroenterology Unit, Federico II University, via Pietro Castellino 141, 80131 Napoli, Italy. Mobile: +39.333.5021090 - Fax: +39.081.7462751.

E-mail: alessa.dalessandro@gmail.com

Key words: Type 2 diabetes mellitus; sitagliptin; saxagliptin; DPP-4 inhibitors.

Contributions: AA, substantial contributions to the conception and design of the work; AD'A, drafting the work and substantial contributions to the acquisition, analysis, and interpretation of data; FPZ, substantial contributions to the acquisition, analysis, and interpretation of data; SN, MG, substantial contributions to the acquisition of data; GD'A, revising it critically for important intellectual content and final approval of the version to be published.

Conflict of interest: the authors declare no potential conflict of interest.

Received for publication: 5 November 2014

Revision received: 12 January 2015.

Accepted for publication: 17 February 2015.

This work is licensed under a Creative Commons Attribution NonCommercial 4.0 License (CC BY-NC 4.0).

(C) Copyright A. Asti et al., 2016

Licensee PAGEPress, Italy

Italian Journal of Medicine 2016; 10:36-41

doi:10.4081/itjm.2016.558 hyperglycemia, due to both a decline of $\beta$-pancreatic insulin production and an increased peripheral insulin resistance. The incidence of this pathology is increasing rapidly, indeed, it was estimated that the number of diabetics will arise from 371 million (2012) to 552 million (2030), representing one of the major challenge to health care worldwide. ${ }^{1}$

Traditional therapies can be still considered suboptimal, since there are no treatments able to guarantee a perfect glycemic control and a stable prevention of diabetic complications, without the risk of hypoglycemia. ${ }^{2}$ For these reasons, the finding that particular hormones, called glucon-like peptide-1 (GLP-1) and glucose-dependent insulinotropic polypeptide, are able to induce a greater secretion of insulin only in the presence of high level of glucose, has quickly attracted the attention of scientists. ${ }^{3,4}$

GLP-1 is a polypeptide, secreted by endocrine cells of small intestine during the digestive phase; the glucose in the intestinal lumen acts as a trigger and induces the release of this hormone. Through the circulation, GLP-1 reaches its receptor on $\beta$ pancreatic cells, stimulating the secretion of insulin in relation to the amount of glucose concentration. Physiologically, the half-life of endogenous GLP-1 is around $2 \mathrm{~min}$; this hormone, indeed, is quickly degraded by a specific peptidase, the dipeptdylpeptidase 4 (DPP-4). ${ }^{5}$

In addition to the stimulus of insulin secretion, the hypoglycemic effect of this substance is enhanced by the inhibition of glucagon release. Moreover, this hormone stimulates the transcription of insulin and 
induces $\beta$-cells neogenesis and proliferation, improving insulin reservoir. ${ }^{6}$

Interestingly, the positive effect of GLP-1 seems not to be limited to glycemic control, ${ }^{5}$ in fact it delays gastric emptying, inducing satiety with a consequent reduction in food intake and it seems to have a cardio-, vaso- and nephroprotective effect. ${ }^{7-10}$

Due to various favorable cardiometabolic and insulinotropic effects, GLP-1 is a very attractive candidate in the management of T2DM. Exogenous administration of GLP-1 analogues (exenatide, liraglutide) and the abatement of degradation by DPP4 inhibitors (saxagliptin, sitagliptin, vildagliptin) are the two approaches used to obtain or maintain high levels of GLP-1. ${ }^{11}$

Sitagliptin and saxagliptin are potent, oral, selective DPP-4 inhibitors. These drugs are able to increase the concentration of endogenous incretin hormones, inducing insulin secretion from $\beta$ cells in a glucose dependent manner. Differences in drug efficacy between these DPP-4 inhibitors have been investigated in few studies, hence the aim of our study is to compare the effectiveness and safety of saxagliptin and sitagliptin in patients who have not achieved adequate glycemic control with metformin.

\section{Materials and Methods}

\section{Characteristics of patients}

Two hundred and fourteen consecutive outpatients with type 2 diabetes mellitus were enrolled. Among them, we selected one hundred and twenty-eight patients, mean age 64.3 years [standard deviation (SD) $\pm 8.4]$, with an average duration of diabetes of 6.5 years $(\mathrm{SD} \pm 4.5)$, who have not achieved an adequate glycemic control while on therapy with maximum dosage of metformin.

We considered as decompensated, patients with glycated hemoglobin $>7.5 \%$ and fasting glucose $(\mathrm{FPG})>140 \mathrm{mg} / \mathrm{dL}$.

\section{Study design}

After the enrollment, patients were randomly divided into two groups: 64 patients $(33 \mathrm{~F}$ and $31 \mathrm{M}$ ) were put on therapy with metformin plus sitagliptin $100 \mathrm{mg} / \mathrm{die}$ and $64(34 \mathrm{~F}$ and $30 \mathrm{M})$ on therapy with metformin plus saxagliptin $5 \mathrm{mg} /$ die (Figure 1).

At time 0' we have detected glycated hemoglobin (HbA1c), FPG, weight, body mass index (BMI), total cholesterol, high- and low-density lipoproteins (HDL and LDL), triglycerides, transaminases and pancreatic amylase. Patients were instructed to maintain their previous eating habits. After four months (T4) all patients were evaluated and the same variables were analyzed. During the follow-up the onset of adverse effects was registered.

\section{Statistical analysis}

Paired Student's $t$-tests were performed to analyze the differences of each group between time 0 and time 4. Unpaired Student's $t$-tests were used to evaluate differences between sitagliptin and saxagliptin subgroups at time 0 and time 4 .

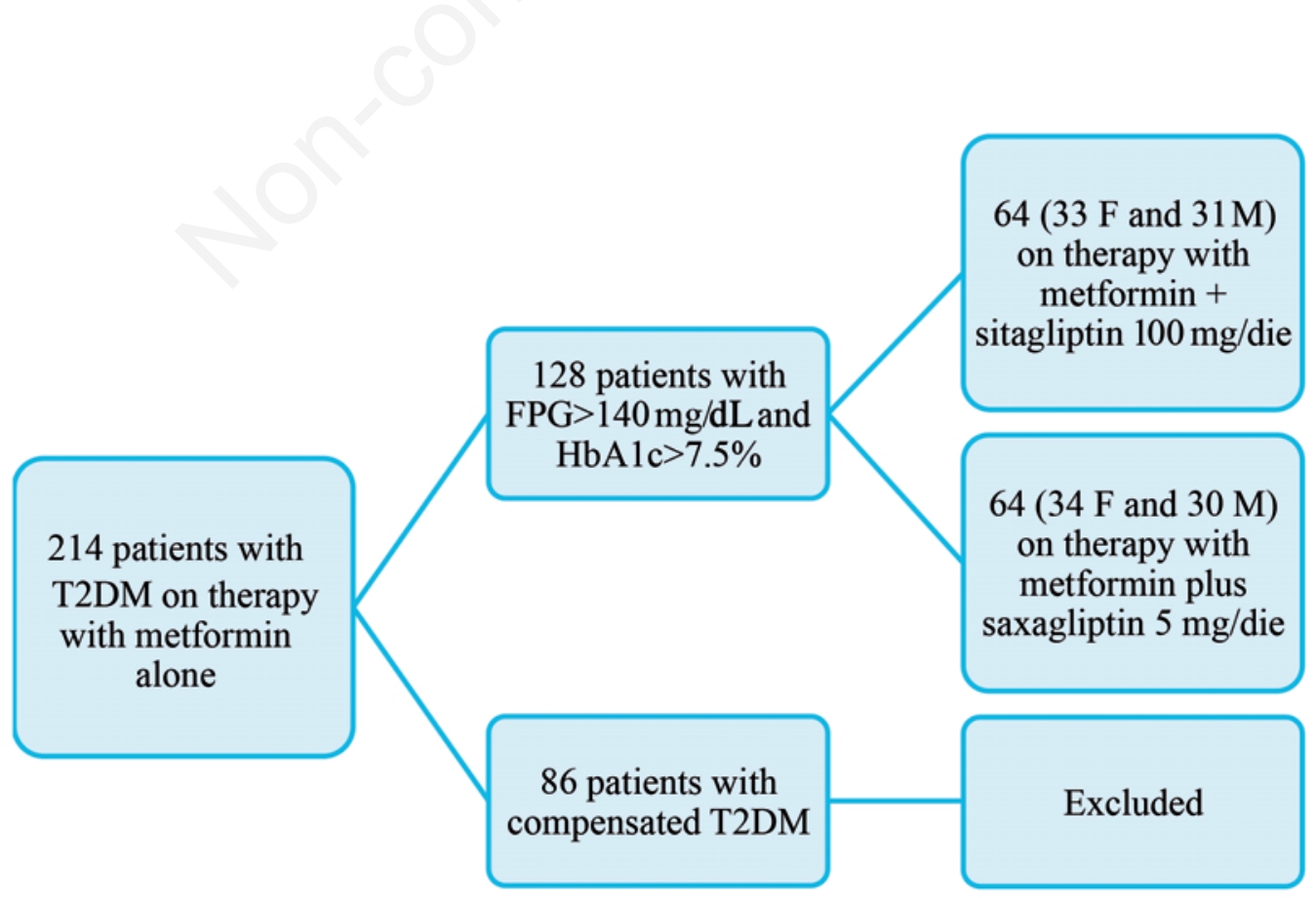

Figure 1. Flow chart demonstrating process of patients' enrollment. FPG, fasting glucose; T2DM, type 2 diabetes mellitus. 


\section{Results}

\section{Sitagliptin}

In sitagliptin group we observed a significant reduction in fasting glucose from $187 \mathrm{mg} / \mathrm{dL}$ to 138 $\mathrm{mg} / \mathrm{dL} \quad(\mathrm{SD} \pm 38 ; \quad \mathrm{P}<0.0001)$ and in glycated hemoglobin from $8.2 \%$ to $7.1 \%$ ( $\mathrm{SD} \pm 1.2 ; \mathrm{P}<0.0001$ ). A significant lowering of weight from $88 \mathrm{~kg}$ to 85.4 ( $\mathrm{SD} \pm 13.9 ; \mathrm{P}<0.0001$ ), $\mathrm{BMI}$ from 32.2 to 31 ( $\mathrm{SD} \pm 3.6$; $\mathrm{P}<0.0001$ ) and triglycerides from $192 \mathrm{mg} / \mathrm{dL}$ to 156 $\mathrm{mg} / \mathrm{dL} \quad(\mathrm{SD} \pm 29.8 ; \mathrm{P}=0.0003)$ was registered, as summarized in Figures 2 and 3. While the reduction in total and LDL cholesterol $(\mathrm{P}=0.3)$ and a slight increase in HDL cholesterol $(\mathrm{P}=0.3)$ did not reach statistical significance.

\section{Saxagliptin}

In saxagliptin group, as shown in Figure 2, we observed a significant reduction in fasting glucose from $191 \mathrm{mg} / \mathrm{dL}$ to $160 \mathrm{mg} / \mathrm{dL}(\mathrm{SD} \pm 31 ; \mathrm{P}<0.0001)$ and in glycated hemoglobin from $8.1 \%$ to $7.5 \%$ $(\mathrm{SD} \pm 1.5 ; \mathrm{P}<0.0001)$. Similarly, as illustrated in Figure

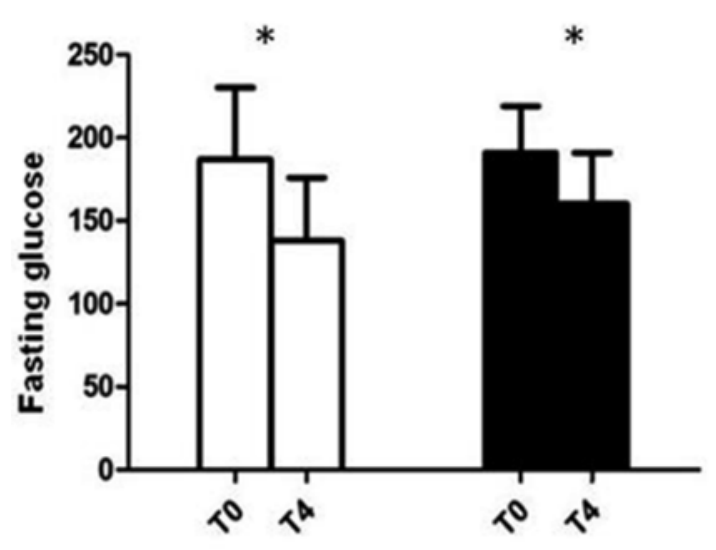

Sitagliptin
Saxagliptin

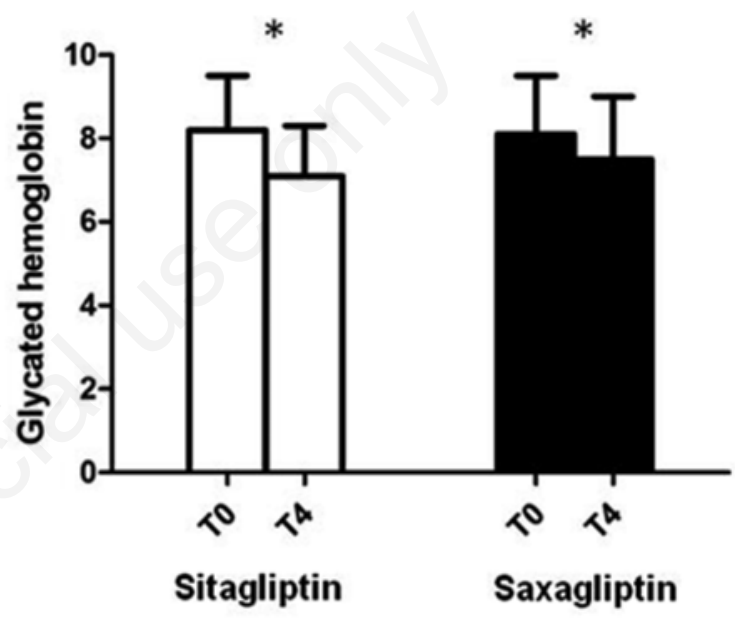

Figure 2. Sitagliptin and saxagliptin have induced a significant reduction of both fasting glucose and glycated hemoglobin in decompensated type 2 diabetes mellitus patients. No differences were found between sitagliptin and saxagliptin subgroups after 4 months. ${ }^{*} \mathrm{P}<0.05$.
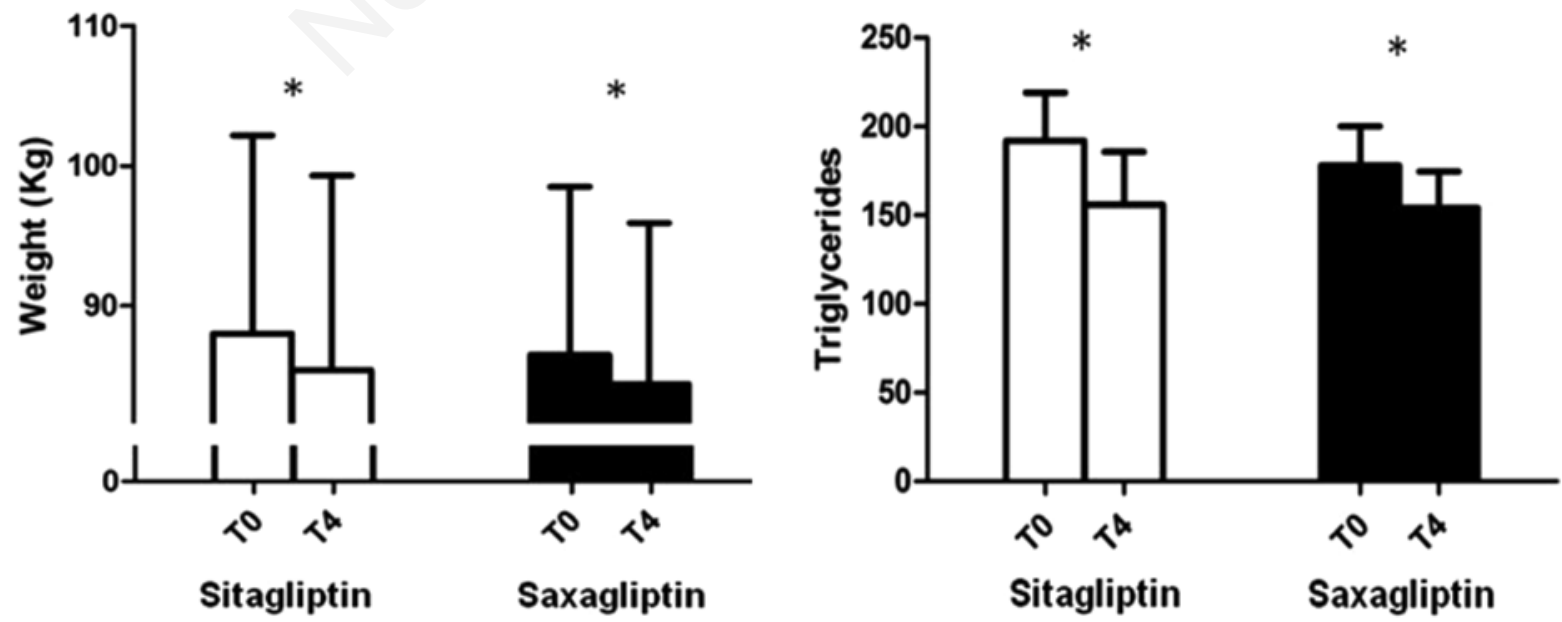

Figure 3. Sitagliptin and saxagliptin have induced a significant weight loss in association with a significant reduction of triglycerides after 4 months of therapy. No differences were found in these parameters between sitagliptin and saxagliptin. $* \mathbf{P}<\mathbf{0 . 0 5}$. 
3, body weight, BMI and tryglicerides showed a significant diminution (from $86.5 \mathrm{~kg}$ to $84.4 \mathrm{~kg}$ with $\mathrm{SD} \pm 11.5 ; \mathrm{P}<0.0001$, from 28 to 27.5 with $\mathrm{SD} \pm 1.4$; $\mathrm{P}<0.0001$ and from $178 \mathrm{mg} / \mathrm{dL}$ to $154 \mathrm{mg} / \mathrm{dL}$ with $\mathrm{SD} \pm 20.6 ; \mathrm{P}=0.0003$, respectively). Also in this subgroup the reduction in total cholesterol, LDL cholesterol $(\mathrm{P}=0.3)$ did not reach statistical significance, HDL cholesterol appeared virtually unchanged $(\mathrm{P}=0.5)$, as well as pancreatic amylase.

\section{Sitagliptin versus saxagliptin}

Sitagliptin and saxagliptin groups, at time 0 , were comparable for mean age, duration of diabetes mellitus, fasting glucose, glycated hemoglobin, cholesterol, tryglicerides, transaminase and pancreatic amylase.

At time 4 , a reduction of $49 \mathrm{mg} / \mathrm{dL}$ in fasting glucose was registered in patients on therapy with sitagliptin, while in saxagliptin group we evaluated a diminution of $31 \mathrm{mg} / \mathrm{dL} \quad(\mathrm{P}>0.05)$. Glycated hemoglobin has shown a reduction of $1.1 \%(\mathrm{SD} \pm 1.2)$ and $0.6 \%(\mathrm{SD} \pm 1.5)$ in sitagliptin and saxagliptin subgroups, respectively $(\mathrm{P}>0.05)$. A similar weight loss was observed in patients taking sitagliptin and saxagliptin $\left(\Delta_{\mathrm{T} 4-\mathrm{TO}}=2.6 \mathrm{~kg}\right.$ and $\Delta_{\mathrm{T} 4 \mathrm{~T} \mathrm{~T}}=2.1 \mathrm{~kg}$, respectively). Also cholesterol, tryglicerides, transaminase and amylase have not shown significant differences between these subgroups.

\section{Adverse effects}

There was no suspension of therapy, adverse events appeared minor and temporary: in sitagliptin group we observed three cases of nausea and one case of nasopharyngitis, which resolved after ten days, in saxagliptin group two cases of nausea and one case of headache which resolved quickly (Table 1).

\section{Discussion and Conclusions}

In this study we have investigated the influence of the DPP-4 inhibitors sitagliptin (5 $\mathrm{mg}$ daily) and saxagliptin (100 mg daily) on glycemic control in decompensated T2DM patients.

Table 1. Description of adverse effects registered during the study.

\begin{tabular}{lcc}
\hline & Saxagliptin & Sitagliptin \\
\hline Headache & 1 & 0 \\
\hline Nausea & 2 & 3 \\
\hline Nasopharyngitis & 0 & 1 \\
\hline Hypersensitivity & 0 & 0 \\
\hline Skin reactions & 0 & 0
\end{tabular}

Our clinical study has demonstrated the efficacy of both saxagliptin and sitagliptin in the achievement of glycemic control in T2DM patients who have not reached this target with metformin alone. In fact, the addition of sitagliptin $5 \mathrm{mg} /$ die induces a reduction in $\mathrm{HbAlc}$ of $1 \%$ (8.2 to $7.1 \%$ ) and a lowering in FPG of $49 \mathrm{mg} / \mathrm{dL}$; obtaining the adequate glycemic control in all patients. Even in the saxagliptin subgroup the glycemic control was reached in all patients, with a reduction in HbA1c of $0.6 \%$ and in FPG of $31 \mathrm{mg} / \mathrm{dL}$.

In line with previous studies, ${ }^{12}$ the addition of either sitagliptin or saxagliptin has shown similar reductions in $\mathrm{HbA} 1 \mathrm{c}$ and $\mathrm{FPG}$, confirming a comparable effectiveness in the treatment of T2DM patients with poor glycemic control with metformin alone.

Similarly, according to our data sitagliptin and saxagliptin share the same effects on lipid profile. In fact, a reduction in triglycerides without a significant lowering of cholesterol levels was demonstrated in both subgroups, likely as effect of a decrease of appetite. To date, a clear association between incretinbased drugs and reduction of BMI was demonstrated only for GLP-1 analogues, while the DPP-4 inhibitors are considered weight-neutral.,13 On the contrary, in this study, after four months of therapy a significant reduction in BMI was registered in patients treated with either saxagliptin or sitagliptin, however a longtern follow-up is necessary to clarify the influence of these drugs on body weight.

Overall, DPP-4 inhibitors are considered welltolerated drugs; the low risk of hypoglycemia depends on the glucose-dependent action of GLP-1. In fact, a key characteristic of this hormone is the ability to induce a reduction in blood glucose concentration only in the presence of hyperglycemia. Different minor adverse effects, including headache, nausea, nasopharyngitis, hypersensitivity and skin reactions, have been described. ${ }^{3,14}$ Elashoff's study also linked incretin-based therapies with pancreatitis and pancreatic cancer in human patients, ${ }^{15,16}$ in our study, although lipases were not detected, no change in pancreatic amylase and no clinical signs of pancreatitis were registered in both groups. The incidence of pancreatic cancer was not assessable due to the short follow-up. In a recent meta-analysis it was demonstrated that DPP-4 inhibitors and metformin combination therapy has a better efficacy than metformin monotherapy, without an increase in any adverse effect, ${ }^{17,18}$ confirming the good safety profile of these drugs. In keeping with this, in our study there was no suspension of therapy and the adverse events appeared minor and temporary. In sitagliptin group we observed three cases of nausea and one case of nasopharyngitis, which resolved after ten days; while in saxagliptin group we detected two cases of nausea 
and one case of headache, which resolved quickly. Although four months are not long enough to establish the long-term safety of these drugs, our data confirmed the high rate of tolerability of these medicaments. Lastly, in the SAVOR-TIMI 53 new data were published on the cardiovascular effects of DPP-4 inhibitors; in this study an higher risk of hospitalization for heart failure in patients on therapy with saxagliptin has been described, without a reduction in ischemic events..$^{19}$ Although we did not evaluated echocardiogram and cardiac biomarkers in our study, no cases of hospitalization for cardiac diseases were observed in both subgroups.

The impressive results achieved with incretinbased therapy have induced a rapid insertion of these drugs in the guidelines. In fact, according to the current American and European treatment algorithms for the management of T2DM, incretin-based therapies are considered, as thiazolidinedione and sulphonylurea, a second-line therapy in combination with metformin in patients with poor glycemic control with diet and metformin monotherapy. ${ }^{20,21}$ Among incretin-based drugs, GLP-1RAs resulted stronger in the reduction of glycemia and BMI than DPP-4 inhibitors, however the oral administration, the lower incidence of gastrointestinal side effects and the minor cost of DPP-4 make them more manageable and costeffective. ${ }^{22-24}$

In conclusion, DPP-4 inhibitors are effective, safe and manageable hypoglycemic drugs, and, considering, the lack of major side effects it is possible to hypothesize they will become the first-choice in the second line therapy for T2DM patients.

\section{References}

1. International Diabetes Federation (IDF). IDF Diabetes atlas. 7th edition; update 2012. Available from: http:// www.idf.org/diabetesatlas

2. Del Prato S. Unlocking the opportunity of tight glycaemic control. Far from goal. Diabetes Obes Metab 2005;7:S1-4

3. Russell-Jones D, Gough S. Recent advances in incretinbased therapies. Clin Endocrinol (Oxf) 2012;77:489-9.

4. Opinto G, Natalicchio A, Marchetti P. Physiology of incretins and loss of incretin effect in type 2 diabetes and obesity. Arch Physiol Biochem 2013;119:170-8.

5. Janssen P, Rotondo A, Mulé F, Tack J. Review article: a comparison of glucagon-like peptides 1 and 2. Aliment Pharmacol Ther 2013;37:18-36.

6. Xiong X, Shao W, Jin T. New insight into the mechanisms underlying the function of the incretin hormone glucagon-like peptide- 1 in pancreatic $\beta$-cells: the involvement of the Wnt signaling pathway effector $\beta$-catenin. Islets 2012;4:359-65.

7. Koole C, Pabreja K, Savage EE, et al. Recent advances in understanding GLP-1R (glucagon-like peptide-1 receptor) function. Biochem Soc Trans 2013;41:172-9.
8. Haluzík M, Frolík J, Rychlík I. Renal effects of DPP-4 inhibitors: a focus on microalbuminuria. Int $\mathrm{J}$ Endocrinol 2013;2013:895102.

9. Ceriello A, Esposito K, Testa R, et al. The possible protective role of glucagon-like peptide 1 on endothelium during the meal and evidence for an "endothelial resistance" to glucagon-like peptide 1 in diabetes. Diabetes Care 2011;34:697-702.

10. Hocher B, Reichetzeder C, Alter ML. Renal and cardiac effects of DPP4 inhibitors-from preclinical development to clinical research. Kidney Blood Press Res 2012;36: 65-84.

11. Aroda VR, Henry RR, Han J, et al. Efficacy of GLP-1 receptor agonists and DPP-4 inhibitors: meta-analysis and systematic review. Clin Ther 2012;34:1247-58.

12. Scheen AJ, Charpentier G, Ostgren CJ, et al. Efficacy and safety of saxagliptin in combination with metformin compared with sitagliptin in combination with metformin in adult patients with type 2 diabetes mellitus. Diabetes Metab Res Rev 2010;26:540-9.

13. Horton ES, Silberman C, Davis KL, Berria R. Weight loss, glycemic control, and changes in cardiovascular biomarkers in patients with type 2 diabetes receiving incretin therapies or insulin in a large cohort database. Diabetes Care 2010;33:1759-65.

14. Ali S, Fonseca V. Saxagliptin overview: special focus on safety and adverse effects. Expert Opin Drug Saf 2013;12:103-9.

15. Butler PC, Elashoff M, Elashoff R, Gale EA. A critical analysis of the clinical use of incretin-based therapies: are the GLP-1 therapies safe? Diabetes Care 2013;36: 2118-25.

16. Labuzek K, Kozłowski M, Szkudłapski D, et al. Incretin-based therapies in the treatment of type 2 diabetes--more than meets the eye? Eur J Intern Med 2013;24:207-12.

17. Fass AD, Gershman JA. Efficacy and safety of dipeptidyl peptidase-4 inhibitors in combination with metformin. Adv Ther 2013;30:337-53.

18. Wu D, Li L, Liu C. Efficacy and safety of dipeptidyl peptidase-4 inhibitors and metformin as initial combination therapy and as monotherapy in patients with type 2 diabetes mellitus: a meta-analysis. Diabetes Obes Metab 2014;16:30-7.

19. Scirica BM, Bhatt DL, Braunwald E, et al. Saxagliptin and cardiovascular outcomes in patients with type 2 diabetes mellitus. N Engl J Med 2013;369:1317-26.

20. Inzucchi SE, Bergenstal RM, Buse JB, et al. Management of hyperglycemia in type 2 diabetes: a patient-centered approach: position statement of the American Diabetes Association (ADA) and the European Association for the Study of Diabetes (EASD). Diabetes Care 2012;35:1364-79.

21. Bailey T. Options for combination therapy in type 2 diabetes: comparison of the ADA/EASD position statement and AACE/ACE algorithm. Am J Med 2013; 126:S10-20.

22. Bergenstal RM, Wysham C, Macconell L, et al. Efficacy and safety of exenatide once weekly versus sitagliptin or pioglitazone as an adjunct to metformin for treatment of type 2 diabetes (DURATION-2): a randomised trial. Lancet 2010;376:431-9.

23. Pratley R, Nauck M, Bailey T, et al. One year of 
liraglutide treatment offers sustained and more effective glycaemic control and weight reduction compared with sitagliptin, both in combination with metformin, in patients with type 2 diabetes: a randomised, parallel-group, open-label trial. Int J Clin Pract 2011;65:397-407.
24. Russell-Jones D, Cuddihy RM, Hanefeld M, et al. Efficacy and safety of exenatide once weekly versus metformin, pioglitazone, and sitagliptin used as monotherapy in drug-naive patients with type 2 diabetes (DURATION-4): a 26-week double-blind study. Diabetes Care 2012;35:252-8. 